\title{
Pregnancy complicated by cardiac disease: fetomaternal outcome
}

\author{
Ankita Singh*, Sudha Prasad \\ Department of Obstetrics and Gynaecology, Lok Nayak Hospital and Maulana Azad Medical College, New Delhi,
} India

Received: 08 January 2018

Accepted: 10 February 2018

\section{*Correspondence:}

Dr. Ankita Singh,

E-mail: drankitasingh@ @otmail.com

Copyright: ( $)$ the author(s), publisher and licensee Medip Academy. This is an open-access article distributed under the terms of the Creative Commons Attribution Non-Commercial License, which permits unrestricted non-commercial use, distribution, and reproduction in any medium, provided the original work is properly cited.

\begin{abstract}
Background: Heart disease complicating pregnancy is an important indirect cause of maternal morbidity and mortality. The study aimed to know the prevalence of heart disease in pregnancy, type of lesions, maternal outcome (obstetric and cardiac) and fetal outcome.

Methods: A retrospective study of 508 women with pregnancy complicated by cardiac disease who delivered at gestation age >28 weeks was carried out at Lok Nayak Hospital associated with Maulana Azad Medical College, New Delhi over a period of 2 years.

Results: Among 508 patients, only 330 were booked cases and 92 were unbooked. 24 patients came to emergency first time with congestive heart failure. Rheumatic heart disease was the predominant lesion seen in $77.5 \%$ cases. Cardiac disease was diagnosed during index pregnancy in $43.5 \%$ cases. 12 cases underwent surgical intervention during pregnancy as they were refractory to medical management. Cesarean section was performed in 109 (21.4\%) cases mainly for obstetric indication. Overall cardiac complication rate was 38.38\%. Maternal mortality was seen in 6 cases. Small for gestation age was seen in $26.4 \%$ cases and preterm labor in $19.9 \%$ cases.

Conclusions: Patients in NYHA I /II have better fetomaternal outcome than in NYHA III/IV. Vaginal delivery is a safe option and caesarean section should be reserved mainly for obstetric indication. Multidisciplinary team approach can significantly improve maternal and fetal outcome.
\end{abstract}

Keywords: Cardiac disease, Fetomaternal outcome, Pregnancy, Retrospective study

\section{INTRODUCTION}

The global incidence of maternal heart disease during pregnancy is around $1 \%$ though regional variation from 0.3-3.5\% has been observed. ${ }^{1-3}$ Pregnancy increases the cardiovascular workload significantly. About $15-52 \%$ of maternal heart disease is first diagnosed during routine antenatal examination or due to deterioration of maternal condition during pregnancy or labour. ${ }^{4}$

Management of cardiac disease during pregnancy is challenging for the obstetrician, cardiologist and neonatologist. The prevalence of rheumatic heart disease in pregnancy has decreased in developed countries but it is still a significant cause of poor fetomaternal outcome in developing countries. The possibility of adverse outcome depends on the type and severity of maternal heart diseases and the extent of functional impairment. Maternal morbidity and mortality depends on the functional classification (New York Heart Association), $0.4 \%$ for New York Heart Association (NYHA) class I and II and $6.8 \%$ for class III and IV. ${ }^{5}$

In India, rheumatic heart disease is the most common cause with Mitral Stenosis (MS) affecting the majority.

Pregnant women with cardiac disease may be divided into: 
- Known case of heart disease under treatment even prior to pregnancy.

- Incidentally diagnosed heart disease during pregnancy.

This retrospective study was carried out to assess the overall fetomaternal status.

The objectives of the present study were to find the prevelance of pregnancy complicated by heart disease during the study period, to evaluate the distribution of heart disease based on etiology and to study the factors affecting fetomaternal outcome in these cases.

\section{METHODS}

This was a retrospective study of 508 pregnant women with cardiac disease conducted over 2 years at Maulana Azad Medical College and associated Lok Nayak Hospital, New Delhi, India. This hospital is a tertiary care center affiliated with GB Pant Hospital, which is a cardiac super speciality where these women are normally followed-up by cardiologist and has expert facilities for cardiothoracic surgery if need arises. Out of 17732 women ( $>=28$ weeks gestation age) delivered at the institute over this period, $508(2.8 \%)$ pregnancies were complicated by heart disease. These included booked and registered cases who were under follow-up as well as unbooked emergency cases. Many of the patients were referred to our institute because of better cardiac care available.

\section{Study design and data collection}

All pregnant women with heart disease were under follow-up of obstetrician as well as cardiologist. The frequency of follow-up varied according to the cardiac status and the duration of gestation. A detailed medical and obstetric history was elicited. Functional class (NYHA) of the patient at the first visit was determined.

Baseline characteristics recorded at the first visit included maternal age, parity, time of diagnosis of heart disease and time of first visit to our hospital, etiology of the underlying cardiac lesion, New York Heart Association (NYHA) functional class, other co-morbidities, cardiac intervention prior to pregnancy or during pregnancy and treatment details of the patient especially anticoagulant therapy.

Routine blood and urine investigations were performed. All patients underwent electrocardiography and echocardiography as a protocol. At each visit, maternal and fetal wellbeing was assessed clinically and any investigation if needed were done.

Pregnancy was monitored till term awaiting spontaneous onset of labour if there was no indication for early delivery. Induction of labour was done using cervigel or concentrated syntocinon. Prophylactic injectable antibiotics were routinely given against infective endocarditis at the onset of labour. Propped up position and intermittent oxygen was given.

Patients were monitored for fluid overload using input /output monitoring and chest auscultation during labour. Outlet forceps or vacuum extractor were used judiciously to shorten second stage of labour. Following delivery, injection frusemide $20 \mathrm{mg}$ was given intravenously.

Appropriate measures were taken to avoid thromboembolism. Women having mechanical prosthesis on anticoagulants were admitted in early pregnancy (6-12 weeks) and again in the third trimester ( $\geq 36$ weeks pregnancy) for switchover from oral anticoagulants to heparin. Heparin was stopped at the onset of labor and restarted $12 \mathrm{hrs}$ after vaginal delivery and $24 \mathrm{hrs}$ after caesarean section. In both cases, warfarin was started 2 days after delivery and overlap was done till International Normalized Ratio (INR) of 2.0 to 3.5 was achieved.

Early ambulation was ensured. Patients remained in hospital for at least 3-5 days postpartum depending on the clinical condition. Postdelivery evaluation and counselling by cardiologist was done with stress on optimisation of cardiac status prior to next pregnancy. Facility for HDU and ICU care was available if needed.

Maternal outcome was analysed on the basis of mode of delivery, presence of co morbidities like: Anaemia, Gestational Diabetes Mellitus, Pregnancy induced hypertension, APH (Placenta Previa and abruption) and Postpartum haemorrhage. Cardiac complications such as pulmonary edema, deterioration in NYHA, arrhythmia were recorded.

Perinatal outcome was assessed according to the incidence of preterm birth ( $<37$ weeks gestation), small for gestational age, perinatal mortality (still birth, intrauterine demise), birth defects, Apgar score, admission to NICU.

\section{Statistical analysis}

Statistical analysis was done using Student's t-test and the $\mathrm{x}^{2}$-test.

\section{RESULTS}

508 women with pregnancy complicated by heart disease were included in the study. The prevalence of cardiac disease was $2.8 \%$ amongst all the deliveries conducted in our hospital during this period. The basic demographic characteristics of the study group are shown in Table 1 .

Most of them were in the age group 18 to 35 years $(96.8 \%)$ and were multigravidas $(61 \%)$. Mean age was $26.45 \pm 3.3$ years. Among them ,330 patients were booked, 86 were registered and rest 92 were unbooked cases. 
Table 1: Baseline characteristics $(n=508)$.

\begin{tabular}{|lll|}
\hline \multicolumn{2}{|l}{ No. } & Percent \\
\hline Age distribution & & \\
\hline$<18$ and $>35$ yrs & 492 & 96.8 \\
\hline Parity & 16 & 3.2 \\
\hline Primigravida & & \\
\hline Multigravida & 198 & 39 \\
\hline Time of first visit & 310 & 61 \\
\hline $1^{\text {st }}$ trimester & & \\
\hline $2^{\text {nd }}$ trimester & 85 & 16.7 \\
\hline 3rd trimester and during labour & 219 & 40.1 \\
\hline Booked & 330 & 43.2 \\
\hline Registered & 86 & 65 \\
\hline Unbooked & 92 & 16.9 \\
\hline When the diagnosis was made & & \\
\hline Not related to pregnancy & 193 & 38 \\
\hline Antenatal & 190 & 37.5 \\
\hline Intra and postpartum & 31 & 6 \\
\hline In previous pregnancy & 94 & 18.5 \\
\hline NYHA class at first visit & & \\
\hline I & 257 & 50.6 \\
\hline II & 140 & 27.5 \\
\hline III & 76 & 15 \\
\hline IV & 35 & 6.9 \\
\hline
\end{tabular}

221(43.5\%) women were diagnosed to have cardiac disease first time during the index pregnancy (either antenatal, intrapartum or postpartum), 287 patients $(56.5 \%)$ were known case of heart disease (diagnosed either at the time of previous pregnancy in 94 patients or not related to pregnancy in 193 cases. Patients got booked in $1^{\text {st }}$ trimester in 85 cases, in $2^{\text {nd }}$ trimester in 204 cases and were seen first time in $3^{\text {rd }}$ trimester or during labour in 219 cases. Most of them were referred from other centres for better maternal and neonatal outcome. Cardiac disease was diagnosed during routine screening in antenatal OPD in 35 patients whereas 24 patients first time came to emergency with congestive heart failure during pregnancy. $257(50.6 \%)$ women were in NYHA class I, $140(27.55 \%)$ in class II,76 (15\%) in class III and $35(6.9 \%)$ in class IV at the time of their first visit to our hospital.

Table 2 shows the distribution of cardiac lesions according to etiology. Rheumatic heart disease was seen in $394(77.5 \%)$ pregnancies. Mitral valve either alone or with other valves was involved in $60 \%$ of them. Mitral stenosis was the commonest lesion (Table 2). Multiple cardiac lesions were seen in 189 (37.2\%) pregnancies.

A total of $64(12.5 \%)$ women had congenital heart disease and atrial septal defect was the commonest lesion seen. Among the miscellaneous lesions, cardiomyopathy constituted the majority (Table 2).
Table 2: Distribution of cardiac lesions.

\begin{tabular}{|lll|}
\hline Etiology & No & Percent \\
\hline Rheumatic heart disease & 394 & $77.55 \%$ \\
\hline MS+MR & 79 & \\
\hline MS & 154 & \\
\hline MS+AS+AR & 21 & \\
\hline MS+AS & 18 & \\
\hline MR & 46 & \\
\hline AS+AR & 21 & \\
\hline MS+TR & 12 & \\
\hline Others & 43 & \\
\hline Congenital heart disease & \\
\hline ASD & 23 & \\
\hline VSD & 18 \\
\hline TOF & 8 \\
\hline PDA & 5 \\
\hline COA & 4 \\
\hline Eisenmenger's syndrome & 4 \\
\hline Others & 2 \\
\hline Miscellaneous & 50 \\
\hline Ischemic heart disease & 5 \\
\hline Arrythmia & 15 \\
\hline DCMP & 25 \\
\hline Others & 5 \\
\hline
\end{tabular}

\section{Maternal obstetric and cardiac outcome}

Maternal outcome during pregnancy is tabulated in Table 3. Anaemia was seen in 101 patients $(19.9 \%)$, pregnancy induced hypertension in 40 patients $(7.8 \%)$, Gestational Diabetes Mellitus in 6 cases $(1.2 \%)$ and antepartum haemorrhage in 15 cases $(2.95 \%)$. 50 patients $(9.8 \%)$ had history of previous caesarean section and $7(1.4 \%)$ of them had twin pregnancy. 25 patients suffered from postpartum haemorrhage $(5 \%)$.

Admission prior to 37 weeks was required in 179 (35.3\%) women either because of obstetric or cardiac indication. Induction of labour was done in 41 patients $(8 \%)$ due to obstetric indication: poor biophysical profile in twelve, intrauterine fetal growth restriction with deranged doppler in nine, oligohydramnios in four, pregnancyinduced hypertension in six, PROM in five and postdatism in five patients. Out of them 35 delivered vaginally and 6 required caesarean section.

Cesarean section was performed in 109 (21.4\%) pregnant women mainly for obstetric indications. 399 (78.6\%) women had vaginal delivery: spontaneous vaginal delivery in $364(71.6 \%)$ cases, assisted breech delivery in four $(0.8 \%)$ and instrumental delivery in 18 cases $(3.5 \%)$. In $<2 \%$ of the cases, patients were taken for caesarean section after consultation with cardiologist due to severe impairment of maternal hemodynamics (in cases of diseases of the aorta, cardiomyopathy with poor ejection fraction and Eisenmenger's syndrome). 
The major cardiac complications are shown in the Table 3. Cardiac disease was diagnosed in the index pregnancy in $43.5 \%$ cases. $43(8.4 \%)$ women had Congestive Heart Failure at least once during this pregnancy. 124 (24.4\%) pregnancies were associated with other cardiac morbidities (arrhythmias in 98, embolism/ hemiparesis/ cerebrovascular accident in 4 and deterioration in NYHA Class in 64 cases).

Table 3: Maternal outcome with cardiac disease.

\begin{tabular}{|lll|}
\hline $\begin{array}{l}\text { Obstetric outcome, c0-morbidities and } \\
\text { complications (n=508) }\end{array}$ & \\
\hline & No. & Percent \\
\hline Anemia & 101 & 20 \\
\hline PIH & 40 & 8 \\
\hline Abruption/APH & 15 & 3 \\
\hline PPH & 25 & 5 \\
\hline GDM & 6 & 1.20 \\
\hline Prev LSCS & 50 & 10 \\
\hline Twin & 7 & 1.40 \\
\hline Cardiac complication & & \\
\hline Pulmonary edema/CHF & 43 & 8.4 \\
\hline Atrial fibrillation/arrythmia & 98 & 19.2 \\
\hline Bacterial endocarditis & 0 & 0 \\
\hline Deterioration of functional class & 64 & 12.6 \\
\hline Thromboembolism & 4 & 0.78 \\
\hline Mortality & 6 & 1.18 \\
\hline Mode of delivery & & \\
\hline Vaginal delivery & 399 & 78.54 \\
\hline caesarean section & 109 & 21.45 \\
\hline Instrumental & 18 & 3.54 \\
\hline & & \\
\hline
\end{tabular}

12 patients had severe MS during current pregnancy and could not be relieved medically. They underwent cardiac intervention during pregnancy (either balloon mitral valvuloplasty or mitral valve replacement). Postoperative period was uneventful, and they tolerated pregnancy well.

There were 6 maternal deaths in the study group. Three women were unbooked, seen first time in labor as an emergency. They suffered from the complications of cardiac failure, pulmonary edema and anemia. Three maternal deaths occurred postnatally, one 3 hours after delivery and 2 others in late postpartum period.

\section{Fetal outcome}

Overall perinatal outcome was good with $97.5 \%$ live birth rate. Details of fetal outcome are summarized in Table 4 . The mean period of gestation at delivery was $37.8 \pm 3.1$ weeks. Of 502 live newborns, 133 babies (26.4 $\%)$ were preterm and 100 babies $(19.9 \%)$ had birth weight $<2.5 \mathrm{~kg}$ though they were term (small for gestational age). Mean birth weight was 2808 $\pm 580 \mathrm{~g}$. Majority of them had Apgar score 9/10 at 5 minutes. Apgar score was less than $9 / 10$ at 5 minutes in $17(3.4 \%)$ babies. $94(18.7 \%)$ required admission to Neonatal intensive care unit (NICU).

There were 31 perinatal lossess ( 7 intrauterine fetal demise, 6 still birth (macerated and live) and 18 neonatal deaths), the perinatal mortality being 6.01\%. 5 (1\%) neonates had congenital malformations: congenital heart disease in two (ASD one, tetralogy of Fallot one), and extracardiac anomalies in three. Both the neonates with cardiac anomalies were born to mothers with rheumatic heart disease.

\section{Cardiac interventions}

174 patients $(34.1 \%)$ had undergone intervention for heart disease prior to pregnancy; of which $93(18.3 \%)$ had mitral valvotomy, $53(10.4 \%)$ had valve replacement and $28(5.5 \%)$ women with Congenital Heart disease underwent corrective surgery. Temporary Pacemaker was placed during pregnancy in 3 cases of Congenital Heart Block. Most of them remained in NYHA I and II.

12 patients required cardiac intervention during the index pregnancy. Post-operative period was uneventful, and they tolerated pregnancy and delivery well.

\section{Effects of cardiac functional class on maternal and fetal outcome}

Table 5 shows the fetomaternal outcome according to NYHA class. Patients in NYHA class III-IV had more maternal and neonatal morbidity and mortality as compared to patients who remained in NYHA I and II. Patients in NYHA class III and IV underwent caesarean section more frequently than those in NYHA Class I and II (38\% vs. 13\%). Small for gestational age babies and prematurity had incidence of $40 \%$ and $32 \%$ respectively in NYHA III and IV, compared with $22 \%$ and $18 \%$ pregnancies where patients were in NYHA class I and II.

Table 4: Fetal outcome in pregnancy with cardiac disease.

\begin{tabular}{|lll|}
\hline & No. & Percent \\
\hline Small for Gestation & 100 & 19.90 \\
\hline Preterm & 133 & 26.4 \\
\hline Still birth & 6 & \\
\hline NICU admission & 94 & 19 \\
\hline Congenital abnormality & 5 & 1 \\
\hline Apgar score $<9 / 10$ & 17 & 3.34 \\
\hline Perinatal mortality & 31 & 6.01 \\
\hline
\end{tabular}

Mean birth weight of babies was $3160 \pm 108 \mathrm{~g}$ among patients who remained in functional classes I and II compared to $2580 \pm 190 \mathrm{~g}$ mean birth weight for patients in functional classes III and IV and this was statistically significant $(\mathrm{p}=0.02)$.

Patients in NYHA class III and IV suffered more cardiac complications (antenatal, intrapartum and postpartum) as 
compared to those with NYHA class I and II (52\% vs. $21 \%)$.

\section{Table 5: Maternal and fetal outcome according to NYHA.}

\begin{tabular}{|lll|}
\hline Outcome parameters & NYHA I \& & NYHA III \\
II $(\%)$ & \& IV (\%) \\
\hline Cardiac complication & 21 & 52 \\
\hline Caesarean section & 13 & 38 \\
\hline Preterm birth & 18 & 32 \\
\hline Low birth weight & 22 & 40 \\
\hline
\end{tabular}

\section{DISCUSSION}

The prevalence of cardiac disease was found to be $2.8 \%$ in the studied population. Rheumatic heart disease was most common cause for cardiac morbidity in pregnant women. Among maternal factors studied, author found that 399 (78.6\%) women had vaginal delivery. Anemia was the most common comorbidity found in association with heart disease. Cardiac complications frequently associated with pregnancy are congestive heart failure and arrhythmia. Among neonatal complications there was a good live birth rate of $97.5 \%$ but $26.4 \%$ were preterm and $19.9 \%$ were small for gestational age.

The main strength of our study is its large sample size; our institute caters to referred cardiac patients from whole of Northern India. Being a tertiary care centre with both obstetric and cardiac superspeciality services in close proximity; full spectrum of fetomaternal presentation and outcome was studied in cardiac patients from preconception upto puerperium.

Present study gives a detailed analysis of pregnancy in cardiac patients but there are certain limitations. Prevalence of cardiac disease at our institute $(2.8 \%)$ may not represent prevalence in general population as our centre caters to referred cardiac patients from all over Northern India. Being a retrospective study, it cannot provide causative factors for cardiac disease but only associated factors. Being a single observer study there was no possible source of bias.

\section{Aetiology}

RHD was 6 times more prevalent than congenital heart disease (Table 2). This data corresponds to other Indian studies where rheumatic valvular disease comprises 69$88 \%$ of all cardiac diseases in pregnancy. ${ }^{6,7}$ In contrast, developed countries have shown marked decline in RHD due to better primary and secondary prevention of streptococcal infections. In present study in $43.2 \%$ of the cases, diagnosis was made first time during the index pregnancy. In a study by Desai et al., in $42 \%$ cases diagnosis was made during pregnancy whereas Hameed et al reported $30.3 \%$ cases of heart disease being diagnosed during index pregnancy. ${ }^{8,9}$ Rate of clinical diagnosis of heart disease was good at our institute due to routine cardiovascular examination during antenatal checkup.

\section{Lesions}

In present study, mitral stenosis was the commonest lesion either alone or with other valvular lesions. Similar distribution was found in studies conducted by Bhatla et al. $^{6}$

Many patients had surgical correction prior to pregnancy and few patients came for preconceptional counseling also. This may be because of our hospital being associated with GB Pant Superspeciality Hospital which is a major referral centre for cardiac disease in North India. Balloon mitral valvotomy and mitral valve replacement were performed in symptomatic patients with severe mitral stenosis preferably in the second trimester of pregnancy which helped the patients tolerate pregnancy well and improve fetal and maternal outcomes. Ideally surgery should be performed pre-pregnancy but in present study no postoperative complications were seen in the surgeries done antenatally.

\section{Maternal obstetric outcome}

Commonest co-morbidities were anemia followed by pregnancy induced hypertension. Both of them increase cardiac workload and can lead to heart failure. Induction of labour was done in $41(8 \%)$ of the women as compared to $39 \%, 4.25 \%$ and $8.4 \%$ in other studies. ${ }^{3,5,10} 35$ out of them delivered vaginally. Caesarean section was done mainly for obstetric indications mainly.

The cardiac indications were coarctation of aorta, severe pulmonary artery hypertension, Eisenmenger's syndrome and cardiomyopathy with severely compromised ejection fraction. In our study, $78.54 \%$ of the women delivered vaginally as compared to $86 \%, 91.42 \%$, and $92 \%$ in other studies. ${ }^{3,9,10}$

\section{Maternal Cardiac Outcome}

The functional class strongly affects the maternal and fetal outcome. $78.2 \%$ of the women in our study were in NYHA class I and II whereas $21.8 \%$ were in class III and IV. These findings were comparable to studies by various authors. ${ }^{1,2,13,14}$

In present study, functional NYHA class worsened in $12.6 \%$ women in the index pregnancy. The postpartum period is a vulnerable period for cardiac patients and risk of complications are high. In present study, seventeen patients developed postpartum pulmonary edema.

Most common contributing factor is increased cardiac load due to increased venous flow through inferior vena cava. Most of them recovered under care of medical intensivist and cardiologist. There were six maternal deaths in the study group. Though patients with NYHA 
III and IV were less in number, they had the worst maternal and perinatal outcome. 4 out of 6 maternal deaths occurred in patients with NYHA class III and IV. ${ }^{4}$ In their study of 486 pregnant patients with rheumatic heart disease, Sawhney et al reported maternal mortality of 10 out of with 8 patients belonged to NYHA III and IV. Subbaiah et al reported that pregnancy with NYHA class III/IV had higher maternal morbidity. They also found that surgical correction in rheumatic heart disease had better outcome when compared to the non-corrected cases. $^{11}$

Overall cardiac complication rate in our study was $38.38 \%$ which is quite high compared to $13 \%$ reported by Siu et al. ${ }^{2}$ However, such magnitude of maternal morbidity is not unexpected when $33.44 \%$ of women were seen as unbooked case in their third trimester of pregnancy or labour.

Therefore, there is further scope for the reduction of maternal mortality and morbidity provided diagnosis is made prenatally and women are counselled and kept under follow-up. Vigilance throughout labor and in the early postpartum period is must to minimize maternal morbidity and mortality.

\section{Fetal Outcome}

An increased incidence of IUGR and low birth weight has been reported in patients with cardiac disease in pregnancy. Proposed explanations include compromised uterine blood supply due to valvular stenosis, maternal arrhythmias and cardioactive drugs including diuretics, digitalis and beta blockers, which alter the hemodynamics. Cervical softening due to hypoxia may increase the chance of preterm delivery. In present study, small for gestational age was seen in $26.4 \%$ as compared to $29.4 \%$ in Barbosa's study. ${ }^{12}$ Preterm labor was seen in $19.9 \%$ in our study whereas in other studies the incidence was $14 \%, 12 \%$ and $23 \% .^{3,9,10}$

Overall, the risk of inheriting congenital heart disease is quoted to be $3-5 \%$ compared to $1 \%$ risk in general population. ${ }^{10}$ In present study, author did not have any inherited neonatal heart disease though congenital heart disease was seen in $12.59 \%$ cases.

Present study highlights that adherence to a carefully conducted antenatal management and delivery protocol allows an uneventful course of pregnancy in most patients. Furthermore, appropriate medical therapy and interventional procedures in the management of cardiac complications permits successful pregnancy even in advanced cases.

Further research is implicated to decrease the burden of rheumatic heart disease which constitutes the main bulk in cardiac pregnant patients. The aim should be primary prevention of rheumatic heart disease.

\section{CONCLUSION}

Rheumatic heart disease is still rampant in India and leads to huge burden on our health care system. From present series author concludes that functional cardiac status during pregnancy influences the maternal and perinatal outcome, the risk being greater in NYHA III and IV. Routine cardiac auscultation by obstetrician during antenatal care can help in diagnosing cases of asymptomtic heart disease. Similarly, physician should be aware regarding screening for rheumatic heart disease especially in females with history of rheumatic fever.

The results of the study suggest that preconceptional counseling, early diagnosis, appropriate referral, surgical correction of cardiac lesions where indicated, maternal and fetal close surveillance during pregnancy and labour with a multidisciplinary team approach comprising of obstetricians, cardiologists, neonatologists and nursing personnel may significantly improve maternal and fetal outcome in pregnancy.

\section{Funding: No funding sources \\ Conflict of interest: None declared \\ Ethical approval: Not required}

\section{REFERENCES}

1. Cox PB, Gogarten W, Marcus MA. Maternal cardiac disease. Curr Opin Anaesthesiol. 2005;18:257-62.

2. Siu SC, Sermer M, Colman JM Alvarez AN, Mercier LA, Morton BC, et al. Prospective multicenter study of pregnancy outcomes in Women with heart disease. Circulation 2001;104:515-21.

3. awhney H, Aggarwal N, Suri V, Vasishta K, Sharma Y, Grover A. Maternal and perinatal outcome in rheumatic heart disease. Int J Gynaecol Obstet. 2003;80(1):9-14.

4. Uebing A, Steer PJ, Yentis SM, Gatzoulis MA. Pregnancy and congenital heart disease. British Medical Journal. 2006;332:401-6.

5. Oron G, Hirsch R, Ben-Haroush A, Hod M, Gilboa $\mathrm{Y}$, Davidi O, et al. Pregnancy outcome in women with heart disease undergoing induction of labour. BJOG: Int J Obstet Gynaecol. 2004;111(7):669-75.

6. Bhatla N1, Lal S, Behera G, Kriplani A, Mittal S, Agarwal N, Talwar KK. Cardiac disease in pregnancy. Int J Gynaecol Obstet. 2003;82(2):153-9.

7. Konar H, Chaudhuri S. Pregnancy Complicated by Maternal Heart Disease: A Review of 281 Women. J Obstet Gynaecol India. 2012;62(3):301-306.

8. Desai DK, Adanlawo M, Naidoo DP, Moodley J, Kleinschmdt I. Mitral stenosis in pregnancy: a four year experience at King Edward VIII Hospital, Durban, South Africa. Br J Obstet Gynaecol 2000;107:953-8.

9. Hameed A, Karaalp IS, Tummala PP, Wani OR, Canetti M, Akhter MW, et al. The effect of valvular heart disease on maternal and fetal outcome of pregnancy. J Am College Cardiol. 2001;37(3):893-9. 
10. Asghar F, Kokab H. Evaluation and outcome of pregnancy complicated by heart disease. J Pak Med Assoc. 2005;55:416-9.

11. Subbaiah M, Sharma V, Kumar S. Heart disease in pregnancy: cardiac and obstetric outcomes. Arch Gynecol Obstet. 2013;(288):23-7.

12. Barbosa PJ, Lopes AA, Feitosa GS, Almeida RV, Silva RM, Brito JC, et al. Prognostic factors of rheumatic mitral stenosis during pregnancy and puerperium. Arq Bras Cardiol 2000;75(3):215-24.
13. Malhotra M, Sharma JB, Tripathii R, Arora P, Arora R. Maternal and fetal outcome in valvular heart disease. Int J Gynecol Obstet. 2004;84(1):11-6.

Cite this article as: Singh A, Prasad S. Pregnancy complicated by cardiac disease: fetomaternal outcome. Int J Reprod Contracept Obstet Gynecol 2018;7:2686-92. 